\title{
CPR flow to prime the ischemic heart during cardiac arrest?
}

BY MARK G ANGELOS, MAHMOOD KHAN

\begin{abstract}
Cardiac arrest is unique among cardiac ischemic syndromes in that all circulation must be generated external to the heart. Although, chest compressions deliver limited blood flow, it may be possible to take advantage of this cardiopulmonary resuscitation (CPR) low-flow state to "prime" the heart in advance of return of restoration of spontaneous circulation. Prior investigation has demonstrated improved cardiac function after perfusing the globally ischemic heart with a cardioprotective agent under low-flow perfusion conditions (modeling CPR flow). These results raise the question as to whether CPR-generated flow can be utilized to induce pharmacological post-conditioning in the arrested heart.
\end{abstract}

Key words: low-flow, GPR, post-conditioning, cardioprotect

\section{Post-conditioning}

There is currently no approved clinical therapy that can be delivered at the onset of reperfusion which will attenuate myocardial reperfusion injury. Recent studies in animal models and in patients undergoing percutaneous coronary intervention (PCI) have demonstrated intermittent short periods of reperfusion interspersed with ischemia prior to full reperfusion to reduce myocardial infarct size and improve functional recovery. $(1,2)$ This reperfusion strategy for the heart, termed post-conditioning, was first described by Zhao et al. (1) and consists of a stuttered on-off ischemia/reperfusion cycle instituted in the first minutes of cardiac 
reperfusion. Post-conditioning activates various cardioprotective mechanisms that reduce myocardial necrosis and improve functional recovery. (2)

The underlying mechanism(s) responsible for post-conditioning are an area of great interest and research. Laboratory studies suggest that the post-conditioning intervention must occur in the first few minutes of reperfusion to be effective. Temporally, this corresponds to the large increase in reactive oxygen species (ROS) generation, observed at the onset of reperfusion. One of the proposed mechanisms for post-conditioning is attenuation of this large ROS burst which occurs at the onset of reperfusion. A reduction in ROS generation and lipid peroxidation has been noted with post-conditioning. (1) In support of a ROS mediated mechanism for post-conditioning, the cardioprotective response is abolished in the presence of a ROS scavenger (N-acetyl cysteine). (3) ROS production seems to be an important trigger for the cardioprotective postconditioning response, but in excess, as occurs with unprotected reperfusion, induces greater cellular injury. (4) In earlier studies, we have noted that low-flow perfusion of the heart attenuates the ROS burst which occurs with the onset of full reperfusion and improves cardiac functional recovery. It appears that classic preand post-conditioning mechanisms activate the survival kinase cascade phosphoinositide 3 kinase (PI3K). The phosphorylation of (PI3K)-Akt in turn activates the cardioprotective reperfusion injury salvage kinase (RISK) pathway. (5) This pathway ultimately leads to downstream inhibition of the mitochondrial permeability pore transition (mPTP). Activation of these pathways has shown significant cardioprotection with both pre- and post-conditioning in prolonged regional ischemia models. (5)

\section{Low Flow generated by cardiopulmonary resuscitation}

As cardiopulmonary resuscitation (CPR) is the only way to initially generate blood flow in the cardiac arrest victim; can this sub-normal flow provide some measure of cardioprotection or attenuation of reperfusion injury following restoration of spontaneous circulation (ROSC)? In several animal studies, controlled reperfusion at $70 \%$ of baseline in ischemic hearts has demonstrated cardioprotection, suggesting a post-conditioning benefit with lower than normal pressure/flow reperfusion. $(6,7)$ This level of flow however, is significantly more than CPR generated flow. The data showing that interventions at the onset of reperfusion, 
such as controlled reperfusion, can improve cardiac outcome, constitutes a powerful argument in support of utilizing perfusion strategies for ameliorating reperfusion injury. Whether such a strategy might be effective during cardiac arrest by CPR generated flow is still unknown. Based on current knowledge, such a strategy must not delay the priority to secure ROSC.

There are as yet no definitive studies to determine whether CPR-generated flow induces cardioprotection. In contrast to regional ischemia, cardiac arrest is a global ischemic insult without means to achieve precise control of reperfusion. Currently the majority of out-of-hospital cardiac arrest patients fail to achieve adequate reperfusion and thereby ROSC. Overall survival to hospital discharge remains low, ranging from 3.0 -16.3\%. (8) During cardiac arrest, chest compressions are instituted to initiate cardiac reperfusion. However, animal studies indicate external chest compressions generate only 1-20\% of normal myocardial blood flow. (9) The benefit of CPR generated low-flow is unclear and may only modestly increase the window of viability. Certainly as the duration of CPR increases, the survival rate decreases. (10) However, there is some earlier evidence from clinical studies that a brief period of CPR may improve survival outcomes. These studies reported that early survival rates were improved if $1.5-3$ minutes of CPR was performed before defibrillation of ventricular fibrillation cardiac arrest patients. $(10,11)$ These data provide evidence that under specific circumstances the limited reperfusion generated by CPR may provide a protective benefit. However, this remains controversial as other studies have shown no benefit. (12) Nevertheless, overall, sudden cardiac arrest victims who receive bystander CPR demonstrate higher rates of survival. (13) The basis for a CPR improved survival benefit is still under investigation.

GPR induced post-conditioning, in which a period of low pressure/flow reperfusion precedes ROSC has been hypothesized, but not tested. (14) It is not clear whether cardioprotective pathways are activated during the low flow of CPR, similar to reports with post-conditioning. Although recent studies have shown benefits of controlled low pressure reperfusion for post-ischemic hearts, (6) the required flow/perfusion pressure to induce protection is much higher than usual CPR-generated flow/pressure. We recently reported in our whole animal rat cardiac arrest model that 10 minutes of CPR preserved cardiac mitochondrial function compared with no-flow cardiac arrest. (15) If low-flow can activate cardioprotective pathways at the onset of reperfusion, further augmentation of these pathways during resuscitation can potentially improve post-arrest 
myocardial recovery.

\section{CPR Delivery of Pharmacologic Cardioprotection}

A second potential strategy to utilize the CPR period is to begin delivery of cardioprotective agents to the heart in advance of the full reperfusion that occurs with ROSC (figure 1). CPR could potentially serve as a delivery mechanism to prime the heart with pharmacologic agents and thereby begin earlier activation of previously described pre- and post-conditioning cardioprotective pathways during the CPR low flow state or at the time of full reperfusion following ROSC. Pharmacologic post-conditioning in the setting of cardiac arrest can potentially improve patient outcome by ameliorating post-resuscitation cardiac dysfunction and injury. However, at present it is not known whether delivery of a pharmacological agent under CPR low-flow conditions induces any cardioprotection at the time of ROSC (full reperfusion). In our recent work, we have used a specific neutrophil elastase inhibitor (Sivelestat), which has shown post-ischemic cardiac protection when delivered at the onset of reperfusion, to test for pharmacologic post-conditioning under low-flow conditions. In this study we utilized the isolated rat heart perfusion model in order to tightly control reperfusion conditions after 25 minutes of global ischemia. Three reperfusion groups were evaluated; 1) full reperfusion $(75 \mathrm{~mm} \mathrm{Hg}$ ) for 60 minutes, 2) reperfusion with low-flow ( $4 \mathrm{ml} / \mathrm{min}$ ) for $3 \mathrm{~min}$ and then full reperfusion for 60 minutes and 3) reperfusion with low-flow (4 ml/min) and Sivelestat $(100 \mu \mathrm{g} / \mathrm{mL})$ for $3 \mathrm{~min}$ and then full reperfusion for 60 minutes. In this model, we noted that low-flow perfusion after global ischemia with sivelestat significantly improved cardiac function and decreased infarct size, despite a 3 minute delay before instituting full reperfusion. (16)

Sivelestat has been approved for use in treatment of acute lung injury in Japan since 2002. (17) It has also been shown in pre-clinical studies to ameliorate ischemia-reperfusion injury in other organs, including the liver, kidneys, and spinal cord. Recently, the cardioprotective effects of sivelestat have been demonstrated in the Langendorff buffer-perfused heart model when administered at the onset of reperfusion. (18) It is plausible to hypothesize that cardioprotection by Sivelestat is associated with both neutrophil-dependent and neutrophil-independent mechanisms, as the drug has demonstrated post- 
ischemic cardioprotection in both blood and Krebs buffer perfused hearts. The existence of a neutrophil-independent mechanism is supported by a study showing that Sivelestat can cause endothelium-independent relaxation of porcine coronary strips. (19) Sivelestat is an ideal pharmacological post-conditioning drug for further investigation under CPR type conditions because of its safety and multi-organ beneficial effects, especially for the heart and lungs.

In clinical practice, the administration of a pharmacologic post-conditioning agent is potentially feasible through a readily placed intravenous catheter in the cardiac arrest victim at the onset of resuscitation. If CPR-generated low-flow can successfully perfuse the heart with pharmacologically active agents, prior to ROSC, this will open up an entirely different pharmacologic approach to treat the cardiac arrest patient. It will enable future resuscitation (and reperfusion) strategies to focus on enhancing cardioprotective signaling pathways during CPR as a strategy to optimize resuscitation of the cardiac arrest patient.

Finally, there are a number of agents which have shown post-conditioning properties when administered at the onset of myocardial reperfusion. This represents yet another potential strategy to improve CPR results by administration of cardioprotective agents at the onset of ROSC. Our group has recently demonstrated that trimetazidine (1-(2,3,4-trimethoxybenzyl) piperazine), which is an anti-ischemic drug $\left(\right.$ Vasterol $\left.^{\circledR}\right)$ provides cardioprotection when administered immediately at the onset of reperfusion. (20) The cardioprotection mediated by trimetazidine appears to be mediated through activation of p38 mitogen-activated protein kinases (MAPK) and Akt signaling.

CPR as a perfusion tool is not an optimal perfusion strategy as it is only a timesensitive temporizing measure until spontaneous circulation can be generated. In addition there is great variability among cardiac arrest subjects, as well as over time in the same subject. Consequently, CPR-generated flow may be too low or variable to reliably activate cardioprotective pathways and instead only add to the ongoing ischemic burden of the heart due to insufficient oxygen and substrate delivery. However, if low-flow induces some cardioprotection either through lowflow itself or by delivery of pharmacologic post-conditioning agents, strategies to augment or amplify these cardioprotective pathways may improve the overall survival of sudden cardiac arrest patients. Such an approach to CPR would constitute an entirely new research direction. 


\section{Conclusions}

The post-conditioning phenomenon has primarily been described in regional cardiac ischemia models and in PCI, under conditions in which reperfusion can be controlled. Unlike the regional ischemia of acute myocardial infarction, controlled reperfusion following cardiac arrest is not possible, particularly in the prehospital environment. Sudden cardiac arrest victims experience global ischemia and are dependent on external chest compressions for initial reperfusion. CPR generated reperfusion is very limited, yields low-flow/perfusion and is insufficient to sustain myocardial viability over time. However, studies indicate better outcome measures if CPR low-flow is started early and in some instances may facilitate successful defibrillation and ROSC. In controlled reperfusion models, we show data suggesting a Sivelestat mediated post-conditioning effect when delivered under low-flow conditions. (16) If CPR generated low-flow can induce cardioprotection either through low-flow itself or by delivery of pharmacologic post-conditioning agents, this would provide a new paradigm for cardiac arrest resuscitation research.

Figure 1. Low-flow pharmacologic conditioning during cardiac arrest.

Resuscitation Strategy utilizing cardiopulmonary resuscitation-generated flow to perfuse the heart with a pharmacologic conditioning agent. Perfusion of the heart would begin in advance of full reperfusion which would occur with restoration of spontaneous circulation (ROSC). Conditioning effects would be manifest as cardioprotection during the post-ROSC reperfusion period.

CPR, cardiopulmonary resuscitation; ROSC, restoration of spontaneous circulation; VF, ventricular fibrillation. 
Figure 1: Low-Flow Pharmacologic Conditioning during Cardiac Arrest

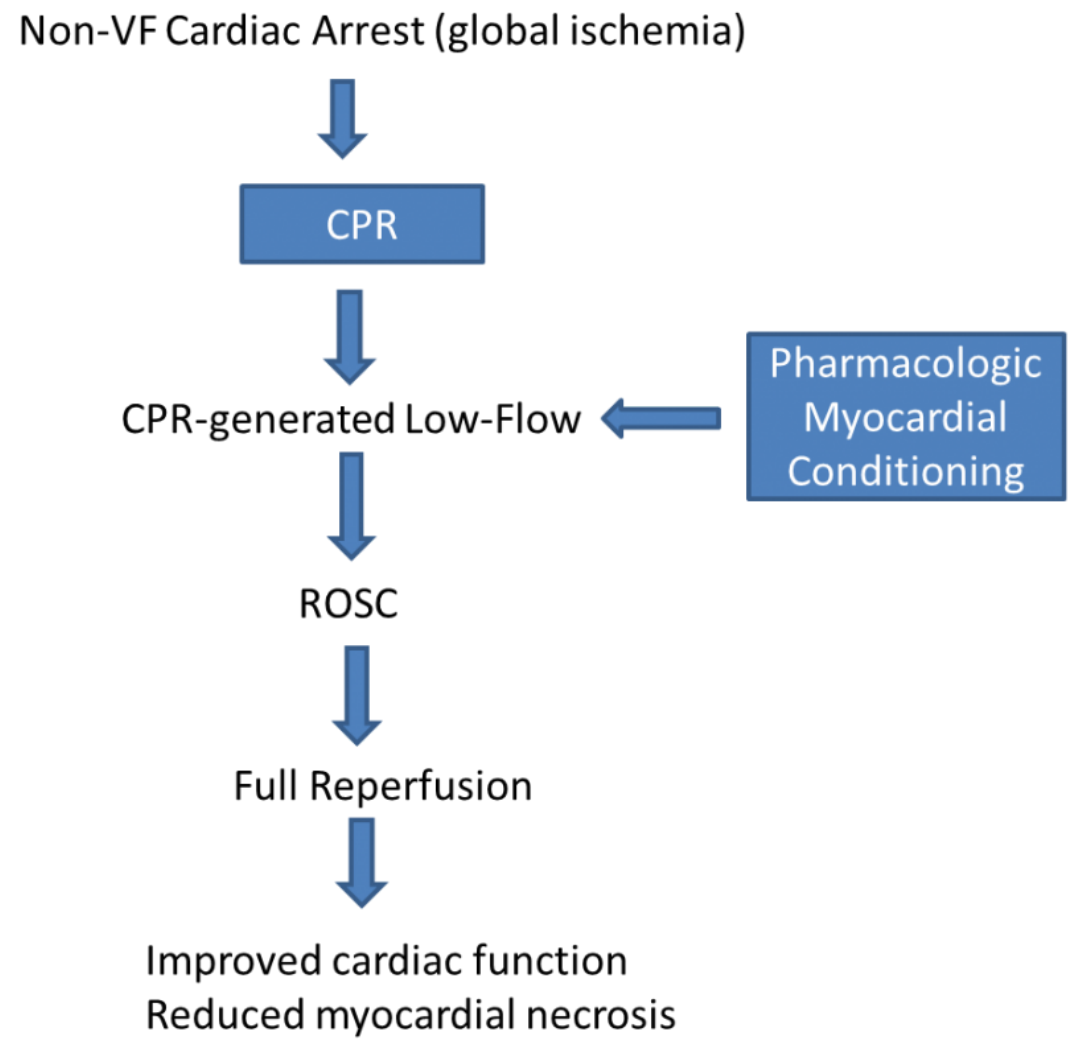

\section{References}

1. Zhao ZQ, Corvera JS, Halkos ME, Kerendi F, Wang NP, Guyton RA, et al. Inhibition of myocardial injury by ischemic postconditioning during reperfusion: comparison with ischemic preconditioning. Am J Physiol Heart Circ Physiol 2003 Aug;285(2):H579-88.

2. Penna C, Mancardi D, Raimondo S, Geuna S, Pagliaro P. The paradigm of postconditioning to protect the heart. J Cell Mol Med 2008 Apr;12(2):435-58.

3. Penna C, Rastaldo R, Mancardi D, Raimondo S, Cappello S, Gattullo D, et al. Postconditioning induced cardioprotection requires signaling through a redoxsensitive mechanism, mitochondrial ATP-sensitive K+ channel and protein kinase $\mathrm{C}$ activation. Basic Res Cardiol 2006 Mar;101(2):180-9.

4. Andreadou I, Iliodromitis EK, Farmakis D, Kremastinos DT. To prevent, protect and save the ischemic heart: antioxidants revisited. Expert Opin Ther Targets 2009 Aug;13(8):945-56. 
5. Hausenloy DJ, Yellon DM. Preconditioning and postconditioning: underlying mechanisms and clinical application. Atherosclerosis 2009 Jun;204(2):334-41.

6. Nemlin C, Benhabbouche S, Bopassa JC, Sebbag L, Ovize M, Ferrera R. Optimal pressure for low pressure controlled reperfusion to efficiently protect ischemic heart: an experimental study in rats. Transplant Proc 2009 Mar;41(2):703-4.

7. Bopassa JC, Michel P, Gateau-Roesch O, Ovize M, Ferrera R. Low-pressure reperfusion alters mitochondrial permeability transition. Am J Physiol Heart Circ Physiol 2005Jun;288(6):H2750-5.

8. Nichol G, Thomas E, Callaway CW, Hedges J, Powell JL, Aufderheide TP, et al. Regional variation in out-of-hospital cardiac arrest incidence and outcome. JAMA 2008 Sep 24;300(12):1423-31.

9. Brown CG, Werman HA, Davis EA, Hobson J, Hamlin RL. The effects of graded doses of epinephrine on regional myocardial blood flow during cardiopulmonary resuscitation in swine. Circulation $1987 \mathrm{Feb} ; 75(2): 491-7$.

10. Bradley SM, Gabriel EE, Aufderheide TP, Barnes R, Christenson J, Davis DP, et al. Survival Increases with CPR by Emergency Medical Services before defibrillation of out-of-hospital ventricular fibrillation or ventricular tachycardia: Observations from the Resuscitation Outcomes Consortium. Resuscitation 2010 Feb;81(2):155-62.

11. Wik L, Hansen TB, Fylling F, Steen T, Vaagenes P, Auestad BH, et al. Delaying defibrillation to give basic cardiopulmonary resuscitation to patients with out-ofhospital ventricular fibrillation: a randomized trial.JAMA 2003 Mar;19;289(11):138995.

12. Jacobs IG, Finn JC, Oxer HF, Jelinek GA. CPR before defibrillation in out-ofhospital cardiac arrest: a randomized trial. Emerg Med Australas 2005 Feb;17(1):3945 .

13. Iwami T, Nichol G, Hiraide A, Hayashi Y, Nishiuchi T, Kajino K, et al. Continuous improvements in "chain of survival" increased survival after out-of-hospital cardiac arrests: a large-scale population-based study. Circulation 2009 Feb 10;119(5):728-34.

14. Zhou Y, Chen D, Ma X, Yang G. Postconditioning in cardiopulmonary resuscitation: a better protocol for cardiopulmonary resuscitation. Med Hypotheses 2009 Sep;73(3):321-3.

15. Yeh ST, Lee HL, Aune SE, Chen CL, Chen YR, Angelos MG. Preservation of mitochondrial function with cardiopulmonary resuscitation in prolonged cardiac arrest in rats. J Mol Cell Cardiol 2009 Dec;47(6):789-97.

16. Aune SE, Yeh ST, Kuppusamy P, Kuppusamy ML, Khan M, Angelos MG. Sivelestat attenuates myocardial reperfusion injury during brief low flow postischemic 
infusion. Oxid Med Cell Longev 2013;2013:279847. doi: 10.1155/2013/279847. Epub; 2013 May 22.:279847.

17. Abe T, Usui A, Oshima H, Akita T, Ueda Y. A pilot randomized study of the neutrophil elastase inhibitor, Sivelestat, in patients undergoing cardiac surgery. Interact Cardiovasc Thorac Surg 2009 Aug;9(2):236-40.

18. Kambe M, Bessho R, Fujii M, Ochi M, Shimizu K. Sivelestat reduces myocardial ischemia and reperfusion injury in rat hearts even when administered after onset of myocardial ischemia. Interact Cardiovasc Thorac Surg 2009Jun;8(6):629-34.

19. Maeda Y, Mitsumizo S, Guo F, Kishi H, Matsuo S, Kobayashi S, et al. Sivelestat relaxes porcine coronary artery via inhibition of $\mathrm{Ca}_{2}+$ sensitization induced by a receptor agonist. J Cardiovasc Pharmacol 2008 May;51(5):476-82.

20. Khan M, Meduru S, Mostafa M, Khan S, Hideg K, Kuppusamy P. Trimetazidine, administered at the onset of reperfusion, ameliorates myocardial dysfunction and injury by activation of p38 MAPK and Akt signaling. J Pharmacol Exp Ther 2010 May;333(2):421-29.

Mark G Angelos, Mahmood Khan Department of Emergency Medicine, Davis Heart Lung Research Institute, The Ohio State University Wexner Medical Center, Columbus, Ohio 43210 Corresponding Author:

Mark G Angelos

Department of Emergency Medicine

The Ohio State University Wexner Medical Center 760 Prior Hall, 376 West 1oth Ave Columbus, Ohio 43210

Phone: 614 293-7536

Fax: 614 293-3124

E-mail: mark.angelos@osumc.edu 